\title{
Analyzing high school students' reasoning about polarization of light
}

\author{
Karolina Matejak Cvenic $\odot,{ }^{1, *}$ Lana Ivanjek, ${ }^{2}$ Maja Planinic $\odot,{ }^{1}$ Katarina Jelicic $\odot,{ }^{1}$ \\ Ana Susac, ${ }^{3}$ and Martin Hopf $\oplus^{4}$ \\ ${ }^{1}$ Department of Physics, Faculty of Science, University of Zagreb, \\ Bijenicka 32, HR-10000 Zagreb, Croatia \\ ${ }^{2}$ Faculty of Physics, Physics Education Research, Technische Universität Dresden, Haeckelstraße 3, \\ 01069 Dresden, Germany \\ ${ }^{3}$ Department of Applied Physics, Faculty of Electrical Engineering and Computing, University of Zagreb, \\ Unska 3, 10000 Zagreb, Croatia \\ ${ }^{4}$ University of Vienna, Austrian Educational Competence Centre Physics, \\ Porzellangasse 4/2/2, 1090 Wien, Austria
}

(Received 7 December 2020; accepted 12 April 2021; published 19 May 2021)

\begin{abstract}
Polarization of light is one of the topics in the Croatian high school physics curriculum. It is taught in the final year of high school education. Twenty-seven Croatian high-school students (aged 18-19 years) were interviewed after regular instruction about their understanding of polarization of light and the model of light itself. This paper reports on the observed students' reasoning strategies and conceptual difficulties. Some of the reported difficulties correspond with previously identified difficulties, but some new difficulties were also found. Students often based their explanations on the remembered but misinterpreted common schematic representations of polarization. Explanations seemed to be created on the spot, suggesting the absence of prior models of polarization.
\end{abstract}

DOI: 10.1103/PhysRevPhysEducRes.17.010136

\section{INTRODUCTION}

Polarization of light is an important phenomenon that has a wide application in everyday life (e.g., polarizing glasses, various screens, 3D movies). Polarization of light is also widely used in industry, for example, to test the strength of the materials or to calculate the density of organic solutions.

In high school physics teaching, interference and diffraction of light are used to demonstrate wave characteristics of light, and polarization of light to show that light is a transverse wave. To understand polarization of light, students must first become familiar with several concepts about light very well:

1. Light can be modeled as an electromagnetic (EM) wave.

2. The electric and magnetic field in the EM wave are interdependent, mutually perpendicular, and constantly oscillating.

3. An EM wave is propagating in a direction that is perpendicular to the directions of the electric and magnetic field oscillations.

\footnotetext{
*karolina@phy.hr

Published by the American Physical Society under the terms of the Creative Commons Attribution 4.0 International license. Further distribution of this work must maintain attribution to the author(s) and the published article's title, journal citation, and DOI.
}

All of this is necessary to cover in teaching before polarization is introduced. Then, students must come to recognize the following features of polarization of light and polarizers ${ }^{1}$ :

4. Unpolarized light consists of numerous EM waves, whose electric fields oscillate in different directions, but all in a plane perpendicular to the propagation direction.

5. When unpolarized light passes through a linear polarizer, it becomes linearly polarized.

6. Linearly polarized light consists of EM waves whose electric fields are oscillating in only one direction.

7. A linear polarizer, used to polarize light, has only one polarizing axis.

8. If the electric field in an EM wave is oscillating parallel to the polarizing axis of the polarizer, the EM wave will pass through the polarizer (both the electric and the magnetic field will pass through).

9. If the electric field in an EM wave is oscillating perpendicularly to the polarizing axis of the

\footnotetext{
${ }^{1}$ There is no consensus about the term for the transparent foil that polarizes the light in the university level physics textbooks. The most common term is polarizer [1-4], but authors used other terms as well, such as polarizing filter [1], polaroid sheet, polaroid filter [2], or polarizing film [3]. The textbooks also mention the Polaroid as a commercial name for the most common polarizer for visible light [1-4]. In this study we decided to use the term polarizer.
} 
polarizer, the EM wave will not pass through the polarizer (neither the electric, nor the magnetic field of the wave).

Additionally, university level students should also know how the polarizing axis of a linear polarizer is achieved on the microscopic level. A linear polarizer consists of long chains of polymer molecules, that are stretched in such a way to make them mutually parallel. The electric field of the incident light will be absorbed by the chains of molecules when the direction of the electric field oscillations is parallel to the chains of molecules. If the electric field of the incident light and the chains of molecules are mutually parallel, the incoming electric field energy will be absorbed by the electrons in polymer molecules of the polarizer, creating an electric current. Considering that the electric field will be completely absorbed by the molecules, neither the electric field parallel to the chains of molecules nor the magnetic field associated with it will pass through the polarizer of that orientation. If the electric field of the incoming light is perpendicular to the chains of molecules, no current will be induced, and both the electric and the magnetic field will pass through the polarizer [3].

Although there exists a body of research on students' difficulties with concepts and phenomena of wave optics [5-10], it mostly concerns the interference and diffraction of light. There is, to our knowledge, no published research on high school students' understanding of polarization of light. Some studies conducted on university students have touched on the issue of polarization, although it was not their primary focus.

A study in the USA found that some university students considered a single slit as a polarizer [6]. Some of them, who treated light as an EM wave with spatial dimensions, claimed that a narrow slit blocks some parts of an EM wave, and others, who treated light as a stream of photons, thought that a narrow slit blocks some photons. To further probe their reasoning, students were asked what they would observe if light was incident on a single narrow vertical slit, after which a polarizer was placed. Some students claimed that the relative orientation of the transmission axis of the polarizer and the vertical side of the slit would determine whether geometrical or wave optics applied [6]. Those students expected only the geometrical image of the slit on the screen if the slit was parallel to the polarizing axis. A similar question was posed to Korean university students and they also seemed to confuse the transmission axis of a polarizer and the vertical side of the narrow slit [11]. Colombo, Jaen, and de Cudmani argued that students have difficulties understanding polarization because all the wave optics phenomena are taught as isolated phenomena and suggested to integrate polarization with other concepts when teaching wave optics [12].

Ambrose et al. concluded that one must have a model of light as an EM wave to reason well about polarization [5]. Many studies showed that the common stationary representation of an EM wave is very problematic for students [5,12-15], and that the 3D nature of an EM wave is not evident to students [5,15]. Several studies investigated students' understanding of EM waves in the context of polarization of light $[5,11,13]$. Students were asked what would happen if a linearly polarized EM wave was incident on a polarizer of a known transmission axis. The results showed that many students did not see the electric and magnetic field in an EM wave as interdependent, because they thought that only the electric or only the magnetic field could pass through the polarizer, depending on the orientation of the transmission axis of the polarizer [5,13]. Ambrose found that some students thought that polarizers have slits (on microscopic level) through which (parts of) the EM wave could pass through [5]. Only a few students in the same study were aware that a polarizer consists of long chains of molecules, but they thought that only light that oscillates parallel to a chain of molecules can pass through the polarizer [5].

Other findings suggest that some students even think that a polarizer does not affect the EM wave at all, meaning that the EM wave passes through the polarizer unchanged [13]. Also, Park and his team found that some students treat polarizers as reducers of intensity only and think that each polarizer placed in front of the incoming light reduces the intensity by the same factor [11].

While there are no studies about high-school students' understanding of polarization, there are several studies about nonuniversity student's understanding of light $[16,17]$. The results showed that participants of different ages (15 years old [16] or 12 to 30 years old [17]) had no coherent models of light and they usually described light as having different forms, like "natural," "electric" or "ultraviolet" [16], or as being "bright" or "colorless" [17].

In Croatia, physics is taught in every year of high schools that prepare students for university (so called gymnasiums) with two or three lessons per week, depending on the type of high school. The physics curriculum in Croatia places polarization in the fourth (final) year of high school, when students are 18-19 years old [18]. Most of the Croatian high school textbooks offer similar approaches to polarization [19-21]. When discussing the polarized and the unpolarized light, the same graphical representation with double-headed arrows is used (Fig. 1). The arrows are said to represent the direction of light's oscillations, or the direction of oscillations of the electric field in an EM wave.

Interestingly, most of the textbooks start with the analogy of the EM wave oscillations with the transversal oscillations of the rope that is passing through the slits of two fences (Fig. 2). If the rope is oscillating in a direction parallel to the longer side of the slits, the rope oscillation will continue even after the second fence. But, if the rope is oscillating in a direction that is perpendicular to the longer side of the slit of the second fence, the rope will not oscillate after that fence. 


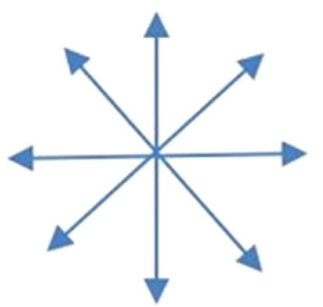

(a)

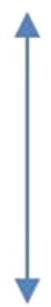

(b)
FIG. 1. Schematic textbook representation of unpolarized light (a) and polarized light (b). The arrows are said to show the direction of the oscillations of light.

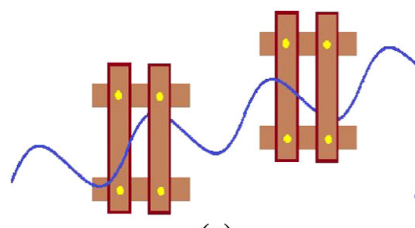

(a)

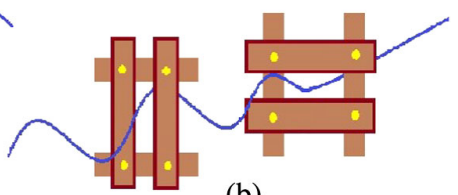

(b)
FIG. 2. Mechanical analogy of polarization of light with an oscillating rope passing through two fences.

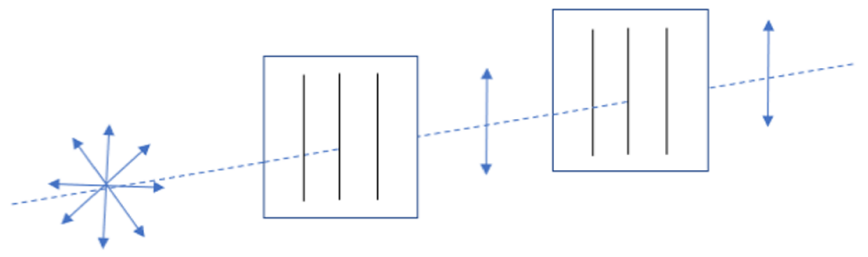

FIG. 3. Schematic representation of one part of the experiment with two polarizers, as typically shown in high school physics textbooks. Vertical lines are representing the polarizing axes of the polarizers.

After that analogy, the linear polarization of light is introduced with an experiment in which two polarizers are used, and their relative orientation is varied (Fig. 3). The unpolarized light passes through the first polarizer and becomes linearly polarized. Depending on the relative orientation of the second polarizer, the polarized light will pass through it completely, partially, or not at all. In some textbooks, the transmission axes on the polarizers are indicated with lines across the polarizers.

It is also mentioned that light can be polarized by reflection and refraction on a transparent medium, which serves as an introduction to the Brewster's law. At the end of the lesson, the polarizing sunglasses are mentioned, and their functioning shortly explained.

The existing research, mostly conducted on university students, demonstrated that polarization of light is a poorly understood topic among university students. Our teaching experience at high school and university level suggests that this topic might be even more difficult for high school students. Therefore, we wanted to investigate how high school students understand this interesting but difficult topic. The research questions this study attempts to answer are the following:

What reasoning strategies and resources do high school students use to explain the polarization of light? What difficulties arise in that process?

\section{THEORETICAL BACKGROUND}

There are two prominent but opposing perspectives regarding knowledge structure coherence: knowledge as theory and knowledge as elements, also known as knowledge-in-pieces [22] Depending on the framework, students' knowledge could be described either as theorylike, quite structured, and coherent, or as consisting of smaller elements that are activated depending on the situation [23]. Knowledge as theory implies that students can approach the explaining of different phenomena within their existing conceptual schema. The problem arises if their knowledge structure is not sufficiently developed to allow them to correctly predict or explain some phenomena, which can lead to cognitive conflict [23]. In this framework cognitive conflict is often used to induce conceptual change although in the reformed approach [24,25] cognitive change does not rely solely on cognitive conflict, a distinction is made between purely intuitive prelearning ideas (preconceptions) and synthetic models that arise from combination of preconceptions and formal teaching, and relative coherence is attributed mostly to preconceptions. The reformed knowledge-as-theory approach embraces some aspects of knowledge in pieces framework (reasoning elements) as a mechanism of generating intuitive ideas.

In knowledge-in-pieces perspective, diSessa [26] argues that students' intuitive physics frameworks consist of numerous fragmented basic reasoning elementshypothetical pieces of knowledge. He called these fragments phenomenological primitives or $p$ prims. They are phenomenological because they are activated when some event needs to be interpreted and they are primitive because they do not need to be explained any further in ones' internal knowledge. They are the most basic level of explanation one can offer based on ones' prior experience [26,27].

DiSessa [27] provided a list of more than $20 p$ prims as a result of his work, but Redish [28] argues that there could be more than a thousand $p$ prims. So, he proposed to use more general reasoning primitives, that combine more elements ( $p$ prims) with everyday items or phenomena.

$p$ prims and some other cognitive structures (concepts, models) are called cognitive resources. Hammer [29] differentiates conceptual and epistemological resources. Conceptual resources are used when students need to explain some physics concept or phenomenon, while 
epistemological resources mostly refer to how students understand knowledge and learning.

Hammer [29] used an interesting analogy between the students' conceptual resources and the smallest bits of programming code (resources in computer science). Programmers could incorporate resources (pieces of programming code) in any program to perform some task. Just like these already written bits of programming code could be used to create new computer programs, people try to solve new problems by relying on their knowledge and strategies that previously solved other problems. If a presented problem is something they are familiar with, people know which of the already existing conceptual resources to use and how to use them, but if the presented problem is new and unfamiliar, people may need to try out a few different resources before reaching a conclusion. Sometimes people will make a mistake by applying a wrong conceptual resource to a certain problem, but that does not mean that the resource itself is wrong.

Hammer argues that the same applies when it comes to students that learn physics. They do not have well-formed conceptions about physical phenomena, but they are not tabulae rasae either. Students gathered knowledge about the real world from their everyday experience.

Hammer and Elby [30] identified resources from which students could build their understanding of physics. Students' raw intuitions provide raw material for building students understanding. Just as resources are neither correct nor incorrect, intuition is also neither correct nor incorrect per se. Resources become (in)correct in their usage. Therefore, students' difficulties in physics result from students' tendency to misapply resources, and the so-called misconceptions that students have may be just robust patterns of misapplication of resources [30]. Students also tend to use simplified reasoning in complex situation that, in combination with activating wrong reasoning elements, may lead to creating wrong answers to physics problems. Students' shortcuts in reasoning were observed by Rozier and Viennot while investigating students' reasoning in thermodynamics [31]. They noticed that students tend to reduce complexity of some thermodynamics problems. To solve a problem, students would either reduce the number of considered variables, or take all variables into account, but in a very simplified way. Such reasoning patterns can be observed in other domains of physics and represent a general students' tendency toward simplified reasoning $[32,33]$.

\section{DATA COLLECTION AND ANALYSIS}

\section{A. The sample}

During the spring of 2018 and the spring of 2019, 27 semistructured interviews with Croatian high school students were conducted. The students were interviewed on several topics of wave optics. All interviewed students were informed about the research by their physics teachers, and they volunteered to participate in the study.

The students in the sample were in the final year of their high school education (age 18-19) and were enrolled in a type of high school called gymnasium in Croatia. Gymnasiums typically prepare students for their future university education and usually attract the academically more able students. At the time of the interview, all the students had completed their regular school instructions on wave optics, that typically lasted for 10 school periods and included among other topics the polarization of light. All the interviewed students took mandatory classes in physics during the previous five and a half years (starting at age 13), with two (or three) physics lessons per week, depending on the type of the school.

In total, 27 students were interviewed (Table I) after their regular school instruction on wave optics. The sample consisted of 13 female and 14 male students. The students came from six different schools. In their 3rd year, 9 students in the study had the grade "good," 10 students had the grade "very good," and 8 students had the grade "excellent" in physics (in Croatia there is a five-level grading system in education: insufficient, sufficient, good, very good, excellent).

\section{B. Interviews}

All interviews were conducted at the Department of Physics, Faculty of Science, University of Zagreb. The interviews probed students' understanding of polarization, interference, and diffraction of light. This paper will focus only on one part of the interview, in which students were asked about the polarization of light. The interviews had the mean duration of $54 \mathrm{~min}$, but the part on polarization of light typically lasted from 5 to $14 \mathrm{~min}$, with the mean time of $9 \mathrm{~min}$. All interviews were audiotaped using smartphone applications (i.e., voice recorder) and later transcribed by the interviewer (K. M. C.).

Two main questions and one experimental task concerning polarization of light were posed to students in each interview:

Question 1: Why are polarized sunglasses recommended to drivers? Why are they better than regular sunglasses? (Students were shown two photos of a car on the road, one taken with and the other without a polarizing filter.)

Experimental task (students were given three equally looking slides containing plastic foil): Here we have three

TABLE I. The number of the students who took part in the study according to their gender and grade.

\begin{tabular}{lccr}
\hline \hline & & 2018 & 2019 \\
\hline Female students: & & 3 & 10 \\
Male students: & 6 & 8 \\
Grades: & Good (average) & 3 & 6 \\
& Very good & 3 & 7 \\
& Excellent & 3 & 5 \\
\hline \hline
\end{tabular}


slides. This one is a polarizer. Of the other two slides, one is a polarizer, and the other one is not. Can you design an experiment to determine which slide is a polarizer?

Question 2: What is polarization of light?

In addition, most of the students were asked to explain their model of light, to better understand their reasoning. Also, they were asked other additional questions too where necessary, i.e., to explain further their statements and/or drawings given with answers to the main questions.

In question 1 we expected the answer that polarized sunglasses remove (partially or completely) the sun glare from some surfaces, such as water, road or glass, because some of the partially polarized reflected light cannot pass through the polarized sunglasses. The regular sunglasses reduce the intensity of the reflected light, but they do not remove the glare. Students were shown two photographs: one of them was taken with a camera containing a polarizer and showed a clear picture of an incoming car. The other was taken without a polarizer and, due to the massive sun glare, the same car was barely visible. Students were asked which photograph was taken with or without a polarizer.

In the experimental task, we expected students to design and perform an experiment. We expected them to take the known polarizer and place one of the unmarked slides in front of it. After that, they should have looked through both slides at the light source and slowly rotated one of the slides. If they had noticed a change in the intensity of the transmitted light with rotation, they should have concluded that the unmarked slide was a polarizer too. If the light intensity had not changed while they were rotating one of the slides, they should have concluded that the unmarked slide was not a polarizer.

As an answer to question 2 at the high school level, we expected students to state that polarization is a wave phenomenon that demonstrates that light is a transverse (EM) wave, and that the phenomenon consists in reducing many directions of oscillations present in unpolarized light to only one when light is passing through a linear polarizer. A complete correct answer to question 2 would also include the discussion of the relative orientation of the electric field of the incident light and the polarizing axis of the polarizer: the incident light would be transmitted if the direction of the electric field oscillations was parallel to the transmission axis of the polarizer, and if the direction of the electric field oscillations was perpendicular to the transmission axis of the polarizer, the incident light could not pass through the polarizer.

Considering that the interviews were audiotaped, students were encouraged to express their thoughts out loud, using the think-aloud technique [34].

Each interviewed student was given a code, that consisted of the letter S, a number 01-27, and an abbreviation for physics grade (E for excellent, VG for very good, $G$ for good).

\section{Analysis}

Transcribed interviews were carefully analyzed by two members of the team independently. The analysis was done using the framework of qualitative content analysis, proposed by Kuckartz [35]. After the analysis, the findings were discussed with other team members. The analysis of the interviews led to the categorization of difficulties or interesting reasoning strategies that the interviewed students expressed or used during the interviews.

The authors first thoroughly read the transcripts to become familiar with the content. Then the transcripts were reread, but this time in search for sentences or parts of the text that would indicate students' strategies and/or resources they used concerning polarization of light. In deciding whether some part of the text should be highlighted as interesting, we were guided by the already identified student difficulties with polarization of light and indications of new difficulties in students' responses.

Parts of the transcripts that seemed to refer to particular student difficulty were highlighted, and those parts were given an initial category label (a descriptive name). Initial category labels that were assigned to some parts of the text, were assigned to other similar parts of the text in that and in the other interviews.

After the initial categorization, all initial categories were grouped and organized thematically. Those groups became the main categories and were given a more general name.

After that, the researchers compared and combined the independently identified categories. The transcripts were reread again, but now, after reading a part of the text that indicated a reasoning strategy or a conceptual difficulty, the more general categories were assigned to that part of the text. The results were then compared and discussed with other team members. In the end we identified three categories that describe students' strategies used on the Experimental task, seven categories on students' difficulties regarding polarization of light and three categories regarding students' model of light.

\section{RESULTS}

The interviewed students were very cooperative during the interviews. They all tried to offer an answer to all questions asked. Of all the interviewed students, only one student did not give any answer to question 2 (what is polarization), and two of the students who were asked about the nature of light, did not offer any answer. Student answers were categorized and grouped according to the expressed experimental or reasoning strategies. In this section, we will first present students' strategies on the experimental task in the interviews, and then student reasoning strategies and difficulties expressed in their answers to questions 1 and 2 and the additional question about the model of light. 


\section{A. Strategies on the experimental task}

Students were asked to design an experiment to find out which of the two unmarked slides was a polarizer. Their strategies in solving this problem were grouped into three categories, labeled E1, E2, and E3.

Students' strategies to determine the unknown polarizer were grouped into three distinctive categories, presented in Fig. 4. For further discussion it was important that students noticed the effect of polarization. Students generally had the idea to look at the light source through the slides, but not always to combine or rotate the slides. If students did not use the correct strategy, it was suggested to them to combine and/or rotate slides.

In the following descriptions of strategies students' quotes are reported as full students' sentences, where it was suitable, or only partially, where that was more appropriate. They are written in italics with quotation marks.

\section{Strategy E1: Looking through two slides at the same time and rotating one of them (9/27 students)}

Nine students (most of them with physics grade excellent) used this correct strategy. They would combine the marked polarizer with one of the unmarked slides and rotate one of them, while looking through both at a light source. If they noticed that the intensity of the light varied with rotating one of the slides, they would conclude that both slides were polarizers. If they noticed no change in intensity while rotating one of the slides, they would repeat the procedure with the remaining slide and correctly identified the unmarked polarizer.

One example of this strategy is given by student S06_E:

"I think that if two polarizers are used and I look through both of them at the same time, that when one [polarizer] is rotated at an angle of $90^{\circ}$ to the other, then one cannot see through it. So, I think this one [is a polarizer]."

One interesting strategy came from the only student with the grade good in this group (S05_G) who wanted to use the calculator as the help for this experiment. He remembered that the calculator screen was polarized, and he wanted to look through the polarizer at the calculator screen because "when you look through one polarizer at the second one, unless they are perfectly aligned, you see nothing." When asked how he could adapt this idea to a situation where he could use only the three given slides, he performed the experiment using the strategy E1.

\begin{tabular}{|c|c|c|c|c|}
\hline Strategy & \multicolumn{2}{|c|}{ Representation of strategy } & \multicolumn{2}{|c|}{$\begin{array}{c}\text { Number of students } \\
\text { and distribution of } \\
\text { grades }\end{array}$} \\
\hline \multirow{4}{*}{$\begin{array}{l}\text { E1. Looking through two slides } \\
\text { at the same time and rotating one } \\
\text { of them }\end{array}$} & & & \multicolumn{2}{|c|}{$9 / 27$ students } \\
\hline & \multirow{3}{*}{ pOLAszer } & & $\mathrm{G}$ & 1 \\
\hline & & & VG & 3 \\
\hline & & & E & 5 \\
\hline \multirow{4}{*}{$\begin{array}{l}\text { E2. Looking at a source of light } \\
\text { through each slide separately } \\
\text { and comparing the observations }\end{array}$} & \multirow{4}{*}{ POLARIZER } & & \multicolumn{2}{|c|}{$16 / 27$ students } \\
\hline & & $\begin{array}{l}\text { UNMARKED } \\
\text { SLUDE }\end{array}$ & $\mathrm{G}$ & 8 \\
\hline & & & VG & 6 \\
\hline & & & E & 2 \\
\hline \multirow{4}{*}{$\begin{array}{l}\text { E3. Looking through two slides } \\
\text { at the same time, but without } \\
\text { rotating them }\end{array}$} & \multirow{4}{*}{\multicolumn{2}{|c|}{ POLARIZER }} & \multicolumn{2}{|c|}{$2 / 27$ students } \\
\hline & & & G & 0 \\
\hline & & & VG & 1 \\
\hline & & & E & 1 \\
\hline
\end{tabular}

FIG. 4. The list and schematic representation of strategies that students used to determine whether the unknown slide was a polarizer and the distribution of students' grades for each strategy. 


\section{Strategy E2: Looking through each slide separately at a source of light and comparing the observations (16/27 students)}

Most of the interviewed students, including the majority of students with grade good (all but one student who used strategy E1), used this strategy. Students would take the marked polarizer in one hand and the unmarked slide in the other one. They would look through only one of them at a time, without rotation, and compare the observations. They mostly described what they saw through the marked polarizer as clean and sharp.

After hearing about the task, student S08_G said that he "might look through both and that he would point them at some light source and look through each one of them [at the light source] and compare the differences and the similarities." After observation, he said that when looking through the polarizer, he "see[s] a clearer picture." Based on that, he concluded (successfully in this case) which slide was the polarizer.

Student S11_G described the experiment with two polarized sunglasses that he remembered from the class (where they combined two polarizing sunglasses and rotated one of them), but he still looked through each slide separately at the light source.

\section{Strategy E3: Looking through two slides at the same time, without rotating them ( $2 / 27$ students)}

Two of the students (grades very good and excellent) combined two slides and looked at the light source through them. They would repeat the procedure with another pair of slides and tried to compare the differences in their observations. During the procedure, they did not rotate any of the slides.

One of them (S27_E) described her strategy before performing the experiment:

S27_E: "So I have some source [of light] and now I have unpolarized light and then there is some polarizer. This is the first one, and then here [after it] ... goes further polarized [light] and then I have this other one [slide which I place behind the first]. Then I do the same thing with this other one [slide] and then I see the differences [between them]."

The strategy started well, but the part with the rotation of the slides was missing. The student said that she was absent from school at the time when this was covered in class, so she did not know how to proceed with the experiment. It was then suggested to her to rotate one of the slides, which helped her to conclude about the second polarizer.

\section{B. Reasoning strategies and difficulties regarding the polarization of light}

In this section the most common students' reasoning strategies and difficulties regarding the polarization of light will be presented. They were found in students' responses to questions 1 and 2, as well as to the additional questions posed during the interviews. Students' answers were carefully analyzed and grouped into seven categories regarding the polarization of light (labeled P1, P2, P3, P4, P5, P6, and P7). In Table II the list of the categories, the number of students who expressed difficulties belonging to each category, as well as the category frequency are listed. The category frequency is the number of explanations that belong to each category. Some students used the same category multiple times during the interview (that is why the category frequency is sometimes bigger than the number of students that used a certain category). Also, it must be noted that categories are not mutually exclusive, meaning that a single answer could sometimes be assigned to multiple categories.

TABLE II. Categories of students' reasoning strategies and resources regarding polarization of light. The correct reasoning category is marked in bold.

\begin{tabular}{|c|c|c|c|c|}
\hline Label & The name of the category & $\begin{array}{l}\text { Num } \\
\text { stude } \\
\text { distrib } \\
\text { their }\end{array}$ & $\begin{array}{l}\text { of } \\
\text { and } \\
n \text { of } \\
\text { des }\end{array}$ & $\begin{array}{l}\text { Category } \\
\text { frequency }\end{array}$ \\
\hline \multirow[t]{4}{*}{ P1 } & \multirow{4}{*}{$\begin{array}{l}\text { Confusing the direction of } \\
\text { oscillation of the electric } \\
\text { field and the direction } \\
\text { of light propagation }\end{array}$} & $9 / 27$ & ents & \multirow{4}{*}{14} \\
\hline & & $\mathrm{G}$ & 2 & \\
\hline & & VG & 4 & \\
\hline & & $\mathrm{E}$ & 3 & \\
\hline \multirow[t]{4}{*}{ P2 } & \multirow{4}{*}{$\begin{array}{l}\text { Polarized light oscillates } \\
\text { in only one direction }\end{array}$} & $5 / 27$ & ents & \multirow{4}{*}{8} \\
\hline & & G & 1 & \\
\hline & & VG & 2 & \\
\hline & & $\mathrm{E}$ & 2 & \\
\hline \multirow[t]{4}{*}{ P3 } & \multirow{4}{*}{$\begin{array}{l}\text { In polarized light the } \\
\text { electric field oscillates } \\
\text { in only one direction }\end{array}$} & $1 / 27$ & ent & \multirow{4}{*}{2} \\
\hline & & $\mathbf{G}$ & $\mathbf{0}$ & \\
\hline & & VG & $\mathbf{0}$ & \\
\hline & & $\mathbf{E}$ & 1 & \\
\hline \multirow[t]{4}{*}{$\mathrm{P} 4$} & \multirow{4}{*}{$\begin{array}{l}\text { Polarization is the } \\
\text { weakening of the light }\end{array}$} & $6 / 27$ & ents & \multirow{4}{*}{8} \\
\hline & & $\mathrm{G}$ & 2 & \\
\hline & & VG & 2 & \\
\hline & & $\mathrm{E}$ & 2 & \\
\hline \multirow[t]{4}{*}{ P5 } & \multirow{4}{*}{$\begin{array}{l}\text { Using geometrical optics } \\
\text { in context of polarization }\end{array}$} & $11 / 27$ & ents & \multirow{4}{*}{18} \\
\hline & & $\mathrm{G}$ & 4 & \\
\hline & & VG & 5 & \\
\hline & & $\mathrm{E}$ & 2 & \\
\hline \multirow[t]{4}{*}{ P6 } & \multirow{4}{*}{$\begin{array}{l}\text { Polarizer as a slit } \\
\text { or a grating }\end{array}$} & $5 / 27$ & ents & \multirow{4}{*}{7} \\
\hline & & $\mathrm{G}$ & 1 & \\
\hline & & VG & 3 & \\
\hline & & $\mathrm{E}$ & 1 & \\
\hline \multirow[t]{4}{*}{ P7 } & Different descriptions & $13 / 27$ & ents & \multirow{4}{*}{21} \\
\hline & of polarization and & $\mathrm{G}$ & 5 & \\
\hline & the functioning & VG & 3 & \\
\hline & of polarizers & $\mathrm{E}$ & 5 & \\
\hline
\end{tabular}




\section{Category P1: Confusing the direction of oscillation of the electric field and the direction of light propagation}

Nine students focused on light propagation while trying to explain polarization. Their explanations could be summarized as follows: the unpolarized light is propagating in all directions and the polarized light is propagating in only one direction. Students who expressed this idea did not mention the oscillations of electric or magnetic field while talking about light (as an EM wave) passing through the polarizer.

Student S03_G, after being asked about the difference between unpolarized and polarized light stated that "[unpolarized] light goes in all directions. It's going everywhere, but polarized light goes in one direction." Student S06_E had the same idea, and he supported his answer with a drawing (Fig. 5). The left-hand side of the image shows unpolarized light, and the right-hand side shows polarized light. He used double-headed arrows to "represent the directions in which light travels." The dart in the middle is signalizing that unpolarized light became polarized light. He said that "after polarization, we have only one direction, or one arrow with 'two heads'." He thought that "through one polarizer passes one direction [of spreading] of light, and when we place another [polarizer], we eliminate that direction too."

Student S24_VG stated that the polarization is the "passing of transverse waves in only one direction" and that the direction of wave propagation is "from the light source to the observer."

Student S26_VG offered a drawing with her explanation of polarization similar to Fig. 1(a), but she misinterpreted the representation of unpolarized light as a point light source emitting light in all directions. She said that the light emitted "from the Sun is not polarized but going in all directions." She drew a point source of light "from where rays of light go in all directions" and said that after the unpolarized light passes through the polarizer it "becomes only one ray [...] that goes in one direction."

\section{Category P2: Polarized light oscillates in only one direction}

To explain or describe polarization of light, some students considered light as a wave. They did not mention

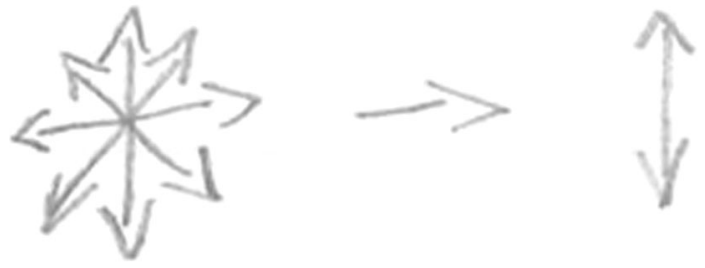

FIG. 5. The drawing of student S06_E. The arrowhead represents the direction of the propagation of light. the nature of that wave (i.e., EM wave). They would say that the unpolarized light is oscillating in all directions, but the polarized light is oscillating in only one direction. Students considered the unpolarized light (even though they did not mention this term) as oscillating in all directions. Student S05_G said that "waves of light oscillate in all directions." He continued that "polarization restricts oscillations only to one, two or how many directions we want." His classmate, student S04_VG, had a similar view of light as "oscillating in all directions." She discussed that when "we use polarizing glasses or filter; the light is directed [to oscillate] in only one direction." She drew an image similar to Fig. 1. She said that "when there is no polarizer, [light is represented] like a "star", but now we have only one [arrow]." This image is the same as the one that student S06_E used (Fig. 5), but the two students interpreted it differently. While S06_E thought that arrows showed the directions of spreading of the light, the S04_VG said the arrows represented the directions of the oscillation of light.

\section{Category P3: In polarized light the electric field oscillates in only one direction}

This category represents the correct view of the polarization of light. Only one student's explanation could be assigned to this category. He discussed the electric field oscillations but did not mention the magnetic field.

While discussing the difference between polarized and regular sunglasses, student S07_E correctly said that the polarized sunglasses "block light of particular polarization." He explained that polarization of light means that "the electric field in an EM wave oscillates only in one direction." Then he supported that with a drawing similar to Fig. 3, where arrows represented "waves that oscillate in different orientations." The polarizer allows only "one direction of light [oscillation]" to pass through. Although the student S07_E also mentioned the oscillations of light, he started his response by saying that the electric field oscillates. So, it can be assumed that by oscillations of light he meant the electric field oscillations.

\section{Category P4: Polarization is the weakening of the light}

The main feature of polarization that students in this category noticed was the change in the intensity of the transmitted light. Six students described polarization only as a process of decreasing the intensity of light, but they did not explain it.

While performing the experimental task (to find the polarizer among unmarked slides with the help of a known polarizer), some students were very focused on the observed intensity of the transmitted light. Some of them, for example, student S12_E, said that "the polarization of light is a phenomenon in which the intensity [of the light] decreases with the help of a polarizer." This statement 
per se is correct but does not capture the essence of polarization. Student S02_VG used the same description of polarization, but he did not use physics terms like intensity, and he referred to the polarizer as a lens: "Polarization is the process of weakening of the light when the light passes through the lens." Throughout the interview, he referred to various optical elements as lenses too. Student S13_E was puzzled with this process of "the weakening of the light." He considered that the polarizers absorb the light in some way, but he could not understand why they absorb light only when rotated to a certain orientation.

This idea was present also in strategy E2 on the experimental task (Fig. 4), where some students, while looking through the polarizer, focused on describing the change of the intensity of the transmitted light and mostly described the light as "somehow dimmed." They remained at the level of description and did not typically offer any explanation of, or the reason for, the reduction of the intensity of light.

\section{Category P5: Using geometrical optics in the context of polarization}

Many students $(11 / 27)$ tried to explain the polarization of light using geometrical optics phenomena, like reflection or refraction of light. Students who used this kind of explanations also represented light as rays. They knew from previous instruction that light is spreading in a straight line through space, and they concluded that if light cannot pass through the polarizer it must have been reflected off or refracted by it.

When asked why we do not see through two polarizers when they are in a certain relative orientation, student S16_G said that "light travels to the last polarizer and then it is reflected from that last one." She drew an image to show what she meant (Fig. 6).

In Fig. 6 light is coming from the source that is located on the left-hand side of the image. Two vertical lines are polarizers, the eye is shown as a curve on the right-hand side. The light is represented as two rays, with arrows that indicate their directions of propagation. Considering that, in a student's view, the light is reflected by the second polarizer, no light reaches the eye.

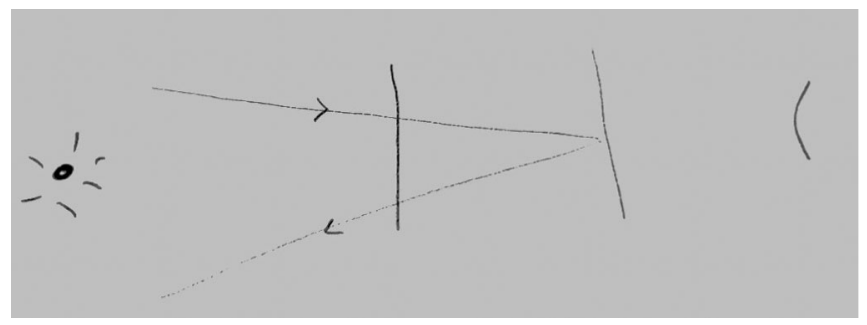

FIG. 6. Drawing that student S16_G made to visualize the polarization of light as the reflection of light off the second polarizer.

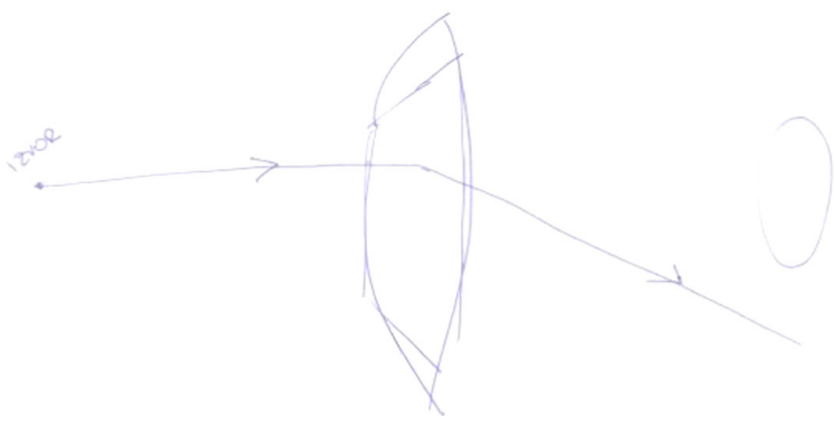

FIG. 7. The drawing of student S20_VG that shows the polarization of light on the sunglasses as the refraction of light. "Izvor" is the Croatian word for the source (of light).

Student S20_VG tried to explain with a drawing how polarizing sunglasses work (Fig. 7). The sunglasses are depicted in the middle of the image. Light is coming from a source marked as a dot on the left-hand side of the image and is represented with a ray that is refracted by the sunglasses. The eye is shown as a circle on the right-hand side of the image.

Student S20_VG said that "when the ray goes through the polarizer then something ... changes. Like, the ray refracts differently." She continued by saying that polarized sunglasses reduce the glare because "somehow the rays refract at some other angle." She concluded that if there were no polarizing glasses "the ray would go straight to the eye."

Student S08_G said that "the rays [of light] are polarized," and he later added that "both light rays and UV rays" could be polarized. He considered that polarized rays "mean that they may not affect so much, something, something else [sight]." This explanation was included into this category because student S08_G used the idea of rays when talking about polarization of light.

\section{Category P6: Polarizer as a slit or a grating}

Five students thought that polarizers have slits on them and that the relative orientation of the slits on the polarizers and the incoming wave determined which waves could pass. A similar view of polarizers as having slits was found in other studies [6]. Student S12_E was explaining the difference between the regular sunglasses and the polarizing sunglasses and said that the polarizing sunglasses have a grating through which waves can pass (on Fig. 8 the grating is indicated by a series of parallel lines on the polarizers). He was showing a sine wave with his hand and said that if the wave was oscillating in some direction and "if one grating is like this [parallel to the direction of wave oscillation] or at some angle, the wave can pass through it. The other is like this [perpendicular to the first] and the wave cannot pass through."

Student S24_VG drew a polarizer similarly as the student S12_E and referred to the parallel lines on the polarizers as "a grating." 


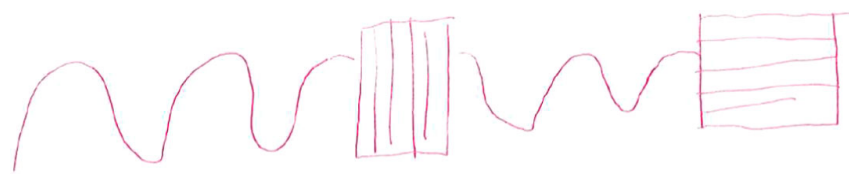

FIG. 8. Student S12_E drew how he imagined that the polarization of light was happening. The parallel lines are "slits" through which the transverse wave can or cannot pass.

\section{Category P7: Different descriptions of polarization and the functioning of polarizers}

This category contains students' views on the polarization itself, and the functioning of polarizers and polarizing sunglasses (7.1.-7.4.).

(a) 7. 1. Partially correct understanding of polarization by reflection. After the first question about the comparison of polarizing glasses and regular sunglasses, students were shown two photographs of the same situation. Even though most of the students could not correctly explain the difference between polarizing and regular sunglasses, all of them determined correctly in which picture the polarizing glasses were used. Only two students offered the correct or partially correct explanation of the situation and one of them recognized some connection with the Brewster's law.

Student S07_E stated that the light reflected from the road was polarized, and he even suggested in what direction the light was polarized.

S07_E: “[On that photograph is used] a vertical polarizer because it blocked all light ... that was horizontally polarized on the road."

SO7_E: "The road is horizontal and when light is reflected then, under specific angle, of course, most of that light will be polarized in that orientation..."

Student S22_E recalled the Brewster's law when asked why the chosen photograph did not show any glare. She explained the Brewster's law and drew the image typically found in textbooks (Fig. 9). She marked the refraction

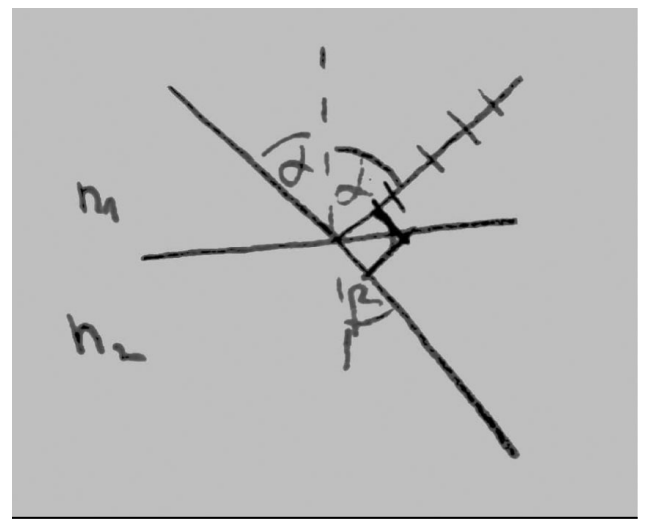

FIG. 9. The schematic representation of Brewster's law, commonly found in textbooks, drawn by student S22_E. indices of the two media, the reflected and the refracted angle, and the $90^{\circ}$ angle between the reflected and the refracted rays. In the beginning she struggled with applying the law to the situation, but in the end, she managed:

\section{S22_E: "So.... it was completely polarized (the reflected light).”}

Although her conclusion was not completely correct (the reflected light was partially polarized) her reasoning went in the right direction.

(b) 7.2. Polarizing glasses block and/or filter light. Six students expressed the idea that polarizing glasses and polarizers block or filter light. Student S08_G thought that polarizing glasses block some parts of light, for example, the UV light.

S08_G: "[Polarization glasses block] UV rays coming to our eyes, so they don't hurt so much or ... that the sun's rays don't affect our eyesight."

Some students referred to polarization as the process of filtering of the light.

Student S23_G, while comparing the regular sunglasses and the polarizing sunglasses said that the regular sunglasses "are not that good because they do not filter sun's rays." When later asked about the polarization of light, he stated:

S23_G: The polarization is segmentation of the light.

IK: What's light segmenting into?

S23_G: Into the waves, I guess. Different [waves].

(c) 7.3. Polarization allows light to distribute differently on various materials. Student S17_VG had an interesting explanation of polarization. He explained that the polarization of light is the process of the more uniform distribution of the light across the polarizer. After looking through a polarizer and a non-polarizer at the light source, he noticed that the observed picture was "clearer" when he was looking through the polarizer. So, he suggested that the polarizer somehow allows light to distribute itself more uniformly across the polarizer.

S17_VG: Polaroid might allow light to be scattered evenly [on its surface] ... so that light is distributed more properly...

IK: Where exactly is light distributed more properly?

S17_VG: On the surface of the glass [on the sunglasses].

IK: What if we have unpolarized light?

S17_VG: Then it mostly goes to the center, where we look [to our eye].

He concluded that the polarization is "a phenomenon that allows light to be distributed differently in some materials [...] to make the image clearer." 
If the light were incident on any transparent polarizing material, he thought it would be evenly distributed across the material making the observed intensity smaller. If light were incident on a non-polarizing material, it would be concentrated in one spot, thus making the intensity greater.

(d) 7.4. Other. Student S03_G treated polarization as a property of some materials, and he also confused functioning of polarizer and derivation of Brewster's law:

S03_G: "The light travels from the source through some, let's say material... glass or crystal that has the property of polarization. I know that one light, how to say, reflects and the other sinks, how to say... Two of them [close the angle] of $90^{\circ}$."

Here he probably described the image typically drawn to introduce Brewster's law, that is commonly found in textbooks (Fig. 9), the light that "sinks" being the refracted ray in the derivation of Brewster's law.

Another student, S10_VG, mixed a lot of concepts and fragments of knowledge together during the interview. Her answers, depending on the question asked, could be sorted in multiple categories. Here, her explanations of polarization will be reported.

While performing the experimental task using strategy E1 (Fig. 4), she referred to the second slide as "the obstacle" the light was encountering and said that there were slits on the polarizers. Her drawing of this experiment is presented in Fig. 10(a). She stated that light was a wave, but the light was represented with rays emerging from the source (drawn as a big yellow spot on the left-hand side of Fig. 10(a)). After passing through the polarizer, the light was represented with a double-headed arrow ("polarized light"). The vertical lines on the polarizers are slits.

A few moments later, while discussing the model of light, she specified that light is a transverse EM wave: "Transverse means it propagates as a sine (curve), and EM means it consists of electronic (her expression) and

(a)

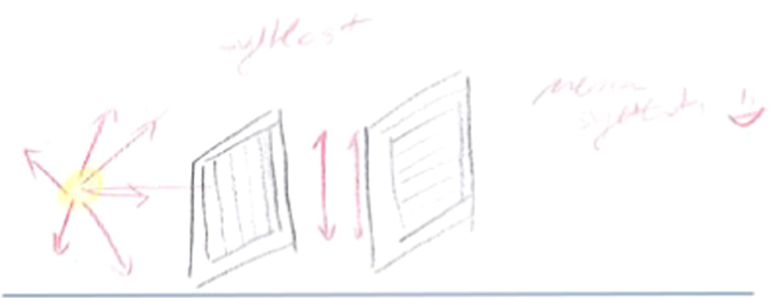

(b)

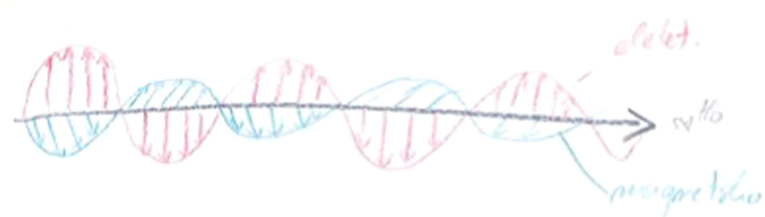

FIG. 10. (a) The drawing of the experiment with two polarizers by student S10_VG. (b) Light as an EM wave. The red curve is an electric field, and the blue curve is the magnetic field. magnetic fields, that are perpendicular." She visualized her statement with a drawing [Fig. 10(b)].

When asked how EM waves are related to polarization, she stated that when electric and magnetic field come to the polarizer "one of them passes through, the other does not. The electric field passes through." This difficulty coincides with the findings of Ambrose and Kesonen [5,13].

This student obviously possesses many fragments of knowledge about light and polarization, but no real model. In a way she is a good example of a conceptual confusion that arises in students when the understanding of the ideas of different models and limits of their applicability are not developed in physics teaching.

\section{Reasoning strategies and difficulties regarding the model of light}

In this section the most common students' reasoning strategies and difficulties regarding their model of light will be presented. that were found in students' responses to question 2, as well as to the additional questions posed during the interviews. Students' answers were grouped into three categories, labeled as L1, L2, and L3 (Table III). Similar to the description of the Table II, some students used the same category multiple times during the interview (the category frequency is sometimes bigger than the number of students that used certain category). Also, the answers from a single student were sometimes sorted into different categories because they sometimes had different views on light.

\section{Category L1: Hybrid models of light}

(a) Confusing wave and particle model of light.Students whose answers were grouped into this category used a combination of wave and particle model to explain what light was. For example, student S02_VG confused the

TABLE III. Categories of students' reasoning strategies and resources regarding the model of light.

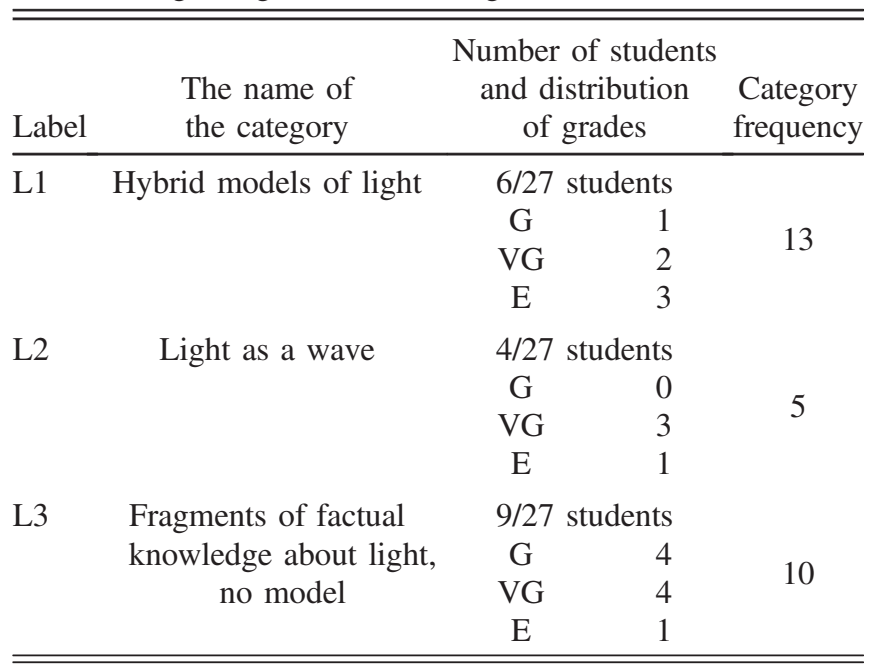


intensity and the wavelength (or frequency) of the radiation, and he claimed that the light "is the EM radiation that does not have high intensity to cause any harm." When asked what differentiates light from other forms of EM radiation, he said that light contains "vast number of little particles that move at the speed of light."

His classmate S03_G also stated that "light is an EM wave where photons are oscillating." He explained that photons "are emitted at the surface of the Sun," and he said that "the EM wave can spread even in vacuum."

Student S27_E expressed her difficulty to differentiate between "light being a wave and light being something...." She "imagine[s] little circles that go up and down, even though [she] think[s] it really isn't like that." When explaining polarization, she said that "particles of light, oscillate in all directions and when they are polarized, they go in only one direction." (This quote is also included in category P2: Explaining polarization with oscillations of light).

(b) L1b: Confusing wave and ray model of light. Student S26_VG expressed a hybrid model of light as a wave and light as a ray. She said that she learned that light is a wave because it behaves like other waves, that it is a transverse wave. But then she tried to connect everything she learned about light and stated that "rays of light are waves in some way." She supported that statement with the example of other wave optics phenomenon, the interference of light:

S26_VG: “... when we were doing interference and all that, then we always thought of it (the light) as waves. But all in all, we ended up working with parallel beams." In her drawing she represented the Sun as the source of light with parallel rays and the waves coming out of it. For her they are basically the same representations of light. It is possible to link rays to wave model of light, as lines that indicate the direction of wave propagation, but it did not seem that students were aware of that.

\section{Category L2: Light as a wave}

Very few students (only four of them) considered light only as a wave.

Student S19_E imagined light as a wave, and she described light as something whose wavelength could be changed. She compared light to sound because both are waves.

S19_E: Well, [light] is some sort of radiation whose wavelength we can change. So, we can see it in different ways, like stronger and weaker. I imagine it like a wave, something like a sound."

She stated that light is a wave, but her explanation of light as "stronger and weaker" indicate that she might have confused some physics quantities, like wavelength and intensity.
Student S24_VG drew a wavy line while talking about light. He perceived light as a transverse wave and differentiated monochromatic and polychromatic light:

S24_VG: "There is a source of light... from where light is coming. It can be monochromatic and polychromatic.

... In polychromatic light there are more colors, our spectrum is bigger."

\section{Category L3: Fragments of factual knowledge about light, no model}

Nine students did not seem to have any model of light, instead they just gave some fragments of factual knowledge about light that they remembered (correctly or incorrectly) from school. Some students treated light as something that enables them to see in some way. One student S21_G was more specific in her answer, saying that we need a source to have light ("The sun gives us light, and because of it we can see.") and that the light "reflects off of other things and that's why we see things." Student S25_G could not give any deeper description or explanation of light, but he knew he cannot see without light ("[Light] is something by which I see everything, and I do not know how to describe it differently.").

Student S14_G said that light is "multiple [rainbow] colors merged into one." Her classmate, S20_VG, stated that light is "a bunch of linearly ordered particles that emit some energy (Fig. 11)."

\section{DISCUSSION}

It seems that most of the students in this sample created their explanations of polarization of light on the spot, combining fragments of their prior knowledge. Perhaps that was best seen when students tried to explain the decrease of the intensity of light after it passed through a polarizer (or when they tried to explain why light was completely blocked by two polarizers adequately combined). This might have activated various conceptual resources they formed in prior learning, for example in geometrical optics, because ten students used geometrical optics reasoning to explain the polarization of light. If light incident on the polarizers did not reach their eyes, they concluded that it must have been either reflected off or refracted by the polarizers. One student concluded that the intensity of the light decreased because the light was uniformly distributed across the polarizer. Other major examples of students' creation of the explanations on the spot are best seen in category P7 (Different descriptions of polarization and the

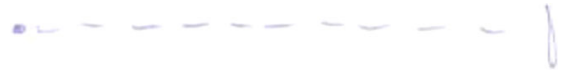

FIG. 11. The representation of light drawn by S20_VG. The black dot at the far left is a light source, and the light is represented with dashed lines (the particles). 
functioning of polarizers), where students combined various unrelated concepts to explain the polarization and the functioning of polarizers.

In the study of Ambrose et al. [6], one of the identified students' difficulties was the misapplication of geometrical and wave optics, where students did not know the domains of applicability of geometrical and wave optics, and thus applied geometrical optics to explain wave optics phenomena and vice versa. The category P5 (Using geometrical optics in the context of polarization) reflects similar findings, that students used phenomena from geometrical optics in wave optics to explain the polarization of light and the functioning of a polarizer.

Activation of resources from geometrical optics might have come also from the misunderstanding of the schematic representation that typically accompanies the derivation of Brewster's law (Fig. 9). As reported in Sec. I, students are taught that light can be polarized either by passing through a polarizer, or by the reflection and refraction on nonmetallic materials. Some students may have simplified and misinterpreted that information and concluded that the polarization of light is due to the reflection or refraction of light on the polarizer also.

While expressing their thoughts or stating their ideas, the interviewed students often produced drawings to explain their ideas. It seems that in a situation where they were discussing the polarization of light, students remembered many schematic representations of polarization from teaching, because some of their drawings are replicas of figures commonly found in textbooks and used by teachers (Figs. 1, 2, 3, 9, and 10b). It appears that the figures used in teaching were a strong visual cue: students often remembered them and then tried to create on the spot an explanation of polarization from them if they did not remember the correct explanation.

Many students based their explanation of polarization of light on Fig. 1. Categories P1 (Confusing the direction of oscillation of the electric field and the direction of light propagation.), P2 (Polarized light oscillates in only one direction), and P3 (In polarized light electric field oscillates in only one direction) emerged from students' interpretation of that figure. For example, student S04_VG interpreted the arrows as directions of the oscillations of the wave (P2), student S07_E interpreted the arrows as directions of the electric field oscillations (P3), but student S06_E interpreted the arrows in a more simplified manner, as indication of the direction of light propagation (P1).

These various interpretations of the same figure could be caused by student's lack of knowledge or understanding of oscillations of electric and magnetic fields in an EM wave, such as light. It also may be an example of students' tendency to simplify abstract and difficult concepts, such as the oscillations of fields in an EM light wave, and replace them with easier concepts, such as the direction of propagation of light (P1) or oscillations of light (P2).
Students whose responses were sorted in $\mathrm{P} 2$ had a vague idea that light is a wave, but they were unsure about what is oscillating in that wave, so perhaps they applied the idea of oscillation to the wave as a whole, possibly imagining it as being similar to the mechanical wave on the rope, something like student S12_E reported and drew in Fig. 8.

Similar difficulties with interpretation of schematic diagrams were found in other studies related to the topic $[5,36]$, and in other domains of physics $[37,38]$.

Elby [39] stated that some visually appealing characteristics of pictures or images can trigger an intuitive knowledge element he called What-You-See-Is-What-You-Get (WYSIWYG). In real life arrows usually symbolize flow or path from one point to the other, and they are often interpreted in the same way by students in more complex or abstract representations (i.e., interpreting electric field lines as paths of motion [40]).

Student S06_E and others, who thought that polarized light propagates in only one direction, activated a good resource which they remembered from the class (after passing through the polarizer, there is only one direction of oscillations in EM (light) wave) but used it partially (only one direction of something is left after the polarizer) because they partially remembered the statement or because they have not understood and have therefore simplified the complicated part. They replaced a difficult concept represented with an arrow (oscillations of electric field in EM wave) with an everyday concept (propagation direction).

Simplification of complex concepts was recognized in various parts of the interviews. Some students replaced the difficult concept of the transmission axis of the polarizer with a simpler concept of a slit or a grating, which may have been also suggested by the fence analogy. But this replacing of concepts might also be due to the activation of WYSIWYG resource because there are lines that symbolize polarizing axes on the schematic representations of the polarizers in some textbooks.

Most of the reasoning categories reported in the Results section originated from students' focus on the intensity of light. Most of the students' answers were the attempts to explain the reduction of the intensity of light by the polarizer, for which various cognitive resources were activated. The change in intensity of light was probably the most striking feature of the experiments with polarizers as well as of the functioning of polarized sunglasses, which explains students' focus on it.

In addition to the drawings that resembled common figures from the textbooks, it is also interesting to notice that some students' statements could be understood as descriptions of those figures. Student S03_G mentioned that "light sinks" during the polarization process. At first, that statement was very puzzling, but his mentioning of the $90^{\circ}$ angle between "the sunken light" and "the reflected light" indicated he might have the derivation of Brewster's 


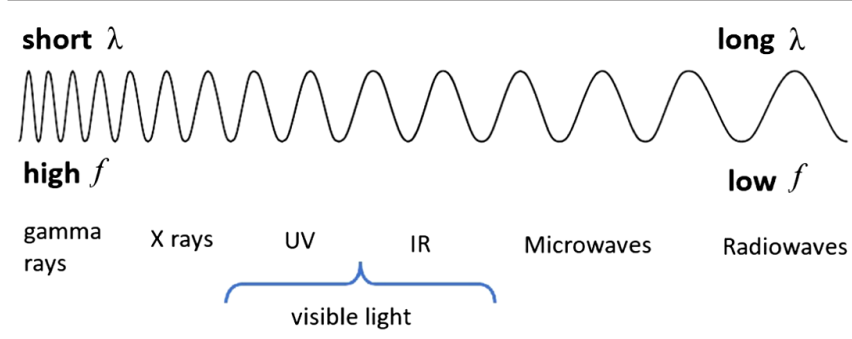

FIG. 12. Schematic representation of the EM spectrum commonly found in textbooks.

law on mind (Fig. 9). Similarly, student S19_E mentioned that light is "some sort of radiation whose wavelength we can change." The "wavelength we can change" could be possibly understood as a description of a figure found in textbooks (Fig. 12), where EM spectrum really is represented as a sine wave with changing wavelength.

Regarding students' models of light, it is interesting to note that the majority of the interviewed students did not use the same model of light while answering the questions about polarization of light and questions about their model of light, meaning that different situations activated different conceptual resources. For example, student S20_VG explained polarization using geometrical optics reasoning (polarization is refraction of light) where she represented light as a ray, but later said that "light is a bunch of linearly ordered particles that emit energy." Student S23_G said that polarization is "segmentation of light to waves" but he later described light as just "a beam of rays." Only six students (out of 20 that were asked about their model of light) used their model of light quite consistently while reasoning about the polarization and when describing light.

\section{CONCLUSIONS AND IMPLICATIONS}

This study investigated how Croatian high school students understand polarization of light after standard physics teaching, what reasoning strategies and resources they use to explain the polarization of light, and what difficulties arise in that process. The existing PER studies on wave optics mostly targeted university students and did not address the polarization of light as their main topics. This study is, to our knowledge, the first one to systematically investigate high school students' understanding of polarization of light.

The findings of this study align well with the knowledge in pieces framework. Students seem to have activated some basic cognitive elements and some resources acquired through teaching to construct explanations of polarization phenomena. It seems that the interviewed students had not formed any firm model of polarization during teaching, so when they were asked to explain polarization, they activated some available resources and tried to produce an explanation (a provisional model) on the spot, on the basis of those resources. The first resource that was activated seemed to have been the schematic representation of polarization seen in class or in a textbook. Then they tried to build an explanation from it, sometimes with other available resources (usually fragments of factual knowledge), and often in a very simplified manner. Similar results suggesting students' misunderstanding of visual material were found in some studies about stationary representations [37-40], and also in studies about students' understanding of dynamic representations [41,42].

When trying to explain polarization, students mostly focused on the reduction of intensity of the light transmitted through the polarizer, which they often tried to explain using resources from geometrical optics. The findings also suggest that students exhibit a considerable confusion concerning the models of light, as some other studies have shown too [7]. Although students' models of light were not in the initial focus of this study, it turned out during the study that this problem underlies the problem of students' poor understanding of polarization. In a very short period of time, students of final years of high school are introduced to three different models of light. First, the light is represented as rays in geometrical optics. Then the light is treated as a wave in wave optics. At the end of the same unit, when discussing the polarization of light, the model of light is broadened once again, and the electromagnetic nature of light is introduced. The units that follow include the unit on photoelectric effect, where light is said to consist of photons.

This leads to the assumption that students do not understand the role of models, or analogies in physics, and that they do not understand their limitations. Different models of light are used to describe different phenomena, just as analogies are used to bridge new and complex phenomena (an EM wave passing through the polarizer) with something simpler (an oscillating rope passing through the slit in the fence). The problem with students not understanding the limitations of models and analogies in physics might lead to confusion when new models or steps in analogies for the same entity are introduced. Students should be made more aware that physics is trying to describe the reality by constructing models and creating analogies.

The schematic representations presented during teaching seemed to have a great impact on students, but it seems that the interpretation of schematic representation did not achieve a similar effect. This could be due to the fact that the concept of the EM wave is very difficult for high school students, and they do not seem to understand it sufficiently. Since student's attention during teaching seems to have been drawn to visually appealing things, it is likely that computer simulations should also be able to catch student's attention. A dynamic simulation of light as an (un)polarized EM wave could be even more visually appealing than static representations, and more informative too. Usually, the textbooks depict the EM wave incident on a polarizer as 
already linearly polarized, probably because representing an unpolarized EM wave is difficult on paper. We feel that this can be overcome with today's technology [14]. In simulations, the incoming light can be represented as an unpolarized wave. However, no matter what is used, the static or dynamic visual representation, our results showed that it is advisable to be very cautious when introducing any representation of complex physics phenomena to students and to probe that they are able to interpret them correctly.

The results of this study show that students emerge from the standard (mostly lecture based) physics teaching in high school without sufficient understanding of polarization and of models of light. In order to overcome some of the reported difficulties we suggest that students should be exposed more to guided inquiry-based teaching, which could help them to familiarize themselves with polarization phenomena, to invent and test explanations and through that process also build better models of phenomena. Polarization is mostly a conceptual physics topic in high school, and it should be probed with many conceptual questions about the interpretation of experiments, both during teaching and on exams. Inducing more of students' discussions of central concepts during teaching could be beneficial for the students and enable teachers to get a better insight in students' understanding and intervene on time to help them form more correct mental models of the phenomenon of polarization.

\section{ACKNOWLEDGMENTS}

This work has been supported in part by Croatian Science Foundations' funding of the Project No. IP-2018-01-9085.
[1] H. D. Young and R. A. Freedman, Sears and Zemansky's University Physics: With Modern Physics (Pearson Education, Upper Saddle River, NJ, 2016).

[2] J. Walker, R. Resnick, and D. Halliday, Halliday \& Resnick Fundamentals of Physics (Wiley, Hoboken, NJ, 2014).

[3] E. Etkina, M. J. Gentile, and A. van Heuvelen, College Physics (Pearson Education, San Francisco, 2014).

[4] E. Hecht, Optics (Addison-Wesley, San Francisco, London, 2002).

[5] B. S. Ambrose, P. R. L. Heron, S. Vokos, and L. C. McDermott, Student understanding of light as an electromagnetic wave: Relating the formalism to physical phenomena, Am. J. Phys. 67, 891 (1999).

[6] B. S. Ambrose, P. S. Shaffer, R. N. Steinberg, and L. C. McDermott, An investigation of student understanding of single-slit diffraction and double-slit interference, Am. J. Phys. 67, 146 (1999).

[7] P. Colin and L. Viennot, Using two models in optics: Students' difficulties and suggestions for teaching, Am. J. Phys. 69, S36 (2001).

[8] L. Maurines, Geometrical reasoning in wave situations: The case of light diffraction and coherent illumination optical imaging, Int. J. Sci. Edu. 32, 1895 (2010).

[9] V. Mešić, E. Hajder, K. Neumann, and N. Erceg, Comparing different approaches to visualizing light waves: An experimental study on teaching wave optics, Phys. Rev. Phys. Educ. Res. 12, 010135 (2016).

[10] K. Wosilait, P. R. L. Heron, P. S. Shaffer, and L. C. McDermott, Addressing student difficulties in applying a wave model to the interference and diffraction of light, Am. J. Phys. 67, S5 (1999).

[11] H. D. Park, K. Kim, M. Kwon, C. Lee, S. Kim, and J. B. Kim, Students' conceptions about polarized electromagnetic waves, in teaching and learning of physics in cultural contexts, in Proceedings of the International Conference on Physics Education in Cultural Contexts, edited by
Y. Park and Y. Park (Cheongwon, South Korea, 2001), pp. 190-196.

[12] E. M. Colombo, M. Jaen, and L. C. de Cudmani, The concept of coherence of learning physical optics, in SPIE Proceedings Vol. 2525, edited by M. J. Soileau (SPIE International Society for Optics and Photonics, Bellingham, WA, 1995), pp. 452-458.

[13] M. H. P. Kesonen, M. A. Asikainen, and P. E. Hirvonen, University students' conceptions of the electric and magnetic fields and their interrelationships, Eur. J. Phys. 32, 521 (2011).

[14] M. Wilson and R. J. Beichner, A comparison of visual representation of E\&M plane waves, in Proceedings of the 2018 Physics Education Research Conference, Washington, D.C., edited by A. Traxler, Y. Cao, and S. Wolf (AIP, New York, 2018).

[15] N. S. Podolefsky and N. D. Finkelstein, Analogical scaffolding and the learning of abstract ideas in physics: An example from electromagnetic waves, Phys. Rev. ST Phys. Educ. Res. 3, 010109 (2007).

[16] D. M. Watts, Student conceptions of light: a case study, Phys. Educ. 20, 183 (1985).

[17] C. Haagen-Schützenhöfer, Students' conceptions on white light and implications for teaching and learning about colour, Phys. Educ. 52, 044003 (2017).

[18] Croatian curriculum (available at https://narodne-novine .nn.hr/clanci/sluzbeni/2019_01_10_210.html).

[19] T. Andreis, M. Plavčić, and N. Simić, Fizika 4, Za 4. razred gimnazija i srodne škole četverogodišnjih programa fizike (Profil, Zagreb, 2007).

[20] D. Horvat and D. Hrupec, Fizika 4, Pojmovi i koncepti. Udžbenik B (Neodidacta, Zagreb, 2010).

[21] J. Labor, Fizika 3, udžbenik za 3. razred gimnazije (Alfa, Zagreb, 2008).

[22] S. Vosniadou, International Handbook of Research on Conceptual Change (Routledge, London, 2008). 
[23] G. Özdemir and D. B. Clark, An overview of conceptual change theories, Eurasia J. Math. Sci. Technol. Educ. 3, 351 (2007).

[24] S. Vosniadou, Reframing the classical approach to conceptual change: Preconceptions, misconceptions and synthetic models in Second International Handbook of Science Education, edited by B. J. Fraser, K. Tobin, and C. J. McRobbie (Springer Netherlands, Dordrecht, 2012), pp. 119-130.

[25] G. J. Posner, K. A. Strike, P. W. Hewson, and W. A. Gertzog, Accommodation of a scientific conception: Toward a theory of conceptual change, Sci. Educ. 66, 211 (1982).

[26] A. A. diSessa, Knowledge in pieces, in Constructivism in the Computer Age, edited by G. Forman and P. Pufall (Lawrence Erlbaum, Hillsdale, NJ, 1988), pp. 49-70.

[27] A. A. diSessa, Toward an epistemology of physics, Cognit. Instr. 10, 105 (1993).

[28] E. F. Redish, A theoretical framework for physics education research: Modeling student thinking, in International School of Physics "Enrico Fermi”, Course; Research on physics education (IOS Press, Amsterdam, 2004), pp 1-64.

[29] D. Hammer, Student resources for learning introductory physics, Am. J. Phys. 68, S52 (2000).

[30] D. Hammer and A. Elby, Tapping epistemological resources for learning physics, J. Learn. Sci. 12, 53 (2003).

[31] S. Rozier and L. Viennot, Students' reasonings in thermodynamics, Int. J. Sci. Educ. 13, 159 (1991).

[32] K. Jelicic, M. Planinic, and G. Planinsic, Analyzing high school students' reasoning about electromagnetic induction, Phys. Rev. Phys. Educ. Res. 13, 010112 (2017).
[33] Laurence Viennot, Reasoning in Physics (Kluwer Academic Publishers, Dordrecht, 2004).

[34] E. Charters, The use of think-aloud methods in qualitative research: An introduction to think-aloud methods, Brock Educ. J. 12, 68 (2003).

[35] U. Kuckartz, Qualitative Text Analysis, A Guide To Methods, Practice \& Using Software (SAGE, Los Angeles, 2014).

[36] P. Colin, F. Chauvet, and L. Viennot, Reading images in optics: Students' difficulties and teachers' views, Int. J. Sci. Educ. 24, 313 (2002).

[37] J. Ametller and R. Pintó, Students' reading of innovative images of energy at secondary school level, Int. J. Sci. Educ. 24, 285 (2002).

[38] F. Stylianidou, Analysis of science textbook pictures about energy and pupils' readings of them, Int. J. Sci. Educ. 24, 257 (2002).

[39] A. Elby, What students' learning of representations tells us about constructivism, J. Math. Behav. 19, 481 (2000).

[40] D. L. Schwartz, Reasoning about the referent of a picture versus reasoning about the picture as the referent: an effect of visual realism, Mem. Cogn. 23, 709 (1995).

[41] V. López and R. Pintó, Identifying secondary-school students' difficulties when reading visual representations displayed in physics simulations, Int. J. Sci. Educ. 39, 1353 (2017).

[42] R. Ploetzner, S. Lippitsch, M. Galmbacher, D. Heuer, and S. Scherrer, Students' difficulties in learning from dynamic visualisations and how they may be overcome, Comput. Hum. Behav. 25, 56 (2009). 